\title{
Corrosion Analysis of Electrical Connectors using SEM
}

\author{
J. Ford ${ }^{1}$, H. Aglan ${ }^{1}$ and A. Ludwick ${ }^{1}$ \\ 1. Tuskegee University, Tuskegee, Al, USA
}

Electrical connectors are critical components that are often designed to perform under harsh environmental weathering conditions. Traditionally designed electrical connectors are usually aluminum with a plated/coated protective layer; these connectors are prone to corrosion and flaking, which cause issues with electrical conductivity, shell degradation, and also affect the electromagnetic interference (EMI) shielding. EMI shielding failure can result in inaccurate or distorted data [1]. Electrical connectors must operate without any conductivity failure, perform without any resistance discrepancy, and be able to mate and unmate during usage.

In this study, two grades of aluminum based Ni-PTFE coated connectors were examined; they are designated Grade-A and Grade-B. The manufacturing processes for these connectors are proprietary. The connectors were tested under salt fog spray. Grade A connector was tested for 500 hours in salt fog and Grade-B connector was tested up to 750 hours in salt fog. Salt fog tests were done in a Q-fog chamber at $35^{\circ} \mathrm{C}$ and $100 \%$ humidity. The electrical connectors were cross sectioned, molded with epoxy, polished, and then examined with a Hitachi S-3400N SEM.

The torque, conductivity, resistance, and corrosion visual observation as functions of connector degradation were evaluated. Grade-A connector disengagement torque and conductivity failed after 500 hours of salt fog with signs of light visual corrosion (spring finger corroded; pits, flaking, and etching). Grade-B connector failed conductivity after 750 hours of salt fog with signs of moderate visual corrosion (discoloration, flaking, etching, and pits).

SEM of Grade-A connector (Figure 1) was performed after 500 hrs of testing but the SEM of Grade-B connector (Figures 2 and 3) was performed after the 750 hours of salt fog testing, when it had failed. Grade-A connector under SEM shows signs of Ni-PTFE coating disbonded from the aluminum base and compromised areas due to corrosion. Grade-B connector under SEM shows signs of corrosion compromising under the Ni-PTFE coating (Figure 2).

SEM images of the different layers of the threads in the Grade -B and Grade -A connectors are shown in Figures $3 \mathrm{a}$ and $3 \mathrm{~b}$ respectively. The thickness of various layers in the outer, inner and external thread were measured for both connectors and are given in Table 1. As can be seen from the measurements, Grade-B after 750 hours of salt fog testing has retained a larger thickness of the electroless layer. The composition of each layer was confirmed using EDX measurements. It is believed that the higher resistance to corrosion of Grade-B connector was the larger thickness of the electroless layer.

References:

[1] Knight, Steven James and Jason E. Anderson. "Corrosion resistant electrical assembly with connectors and multi-port junction block." U.S. Patent No. 8,888,535. 18 Nov. 2014. 

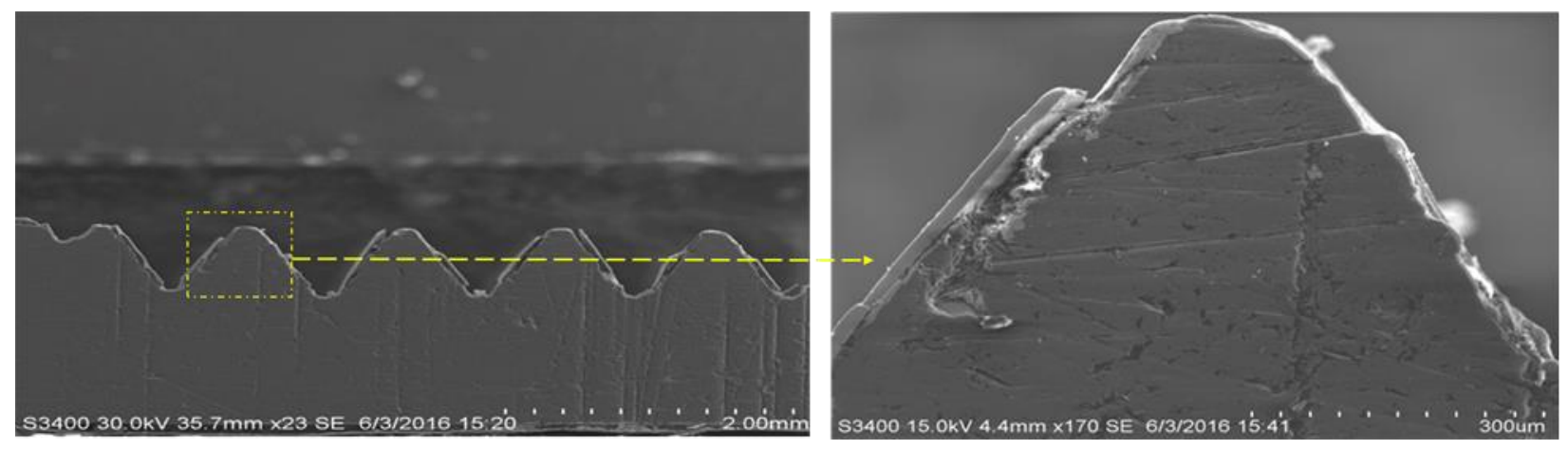

Fig

ure

1.

SE

$M$

of

de-A Ni-PTFE connector viewed from the outer tooth. The picture on the right is at a higher

Gra
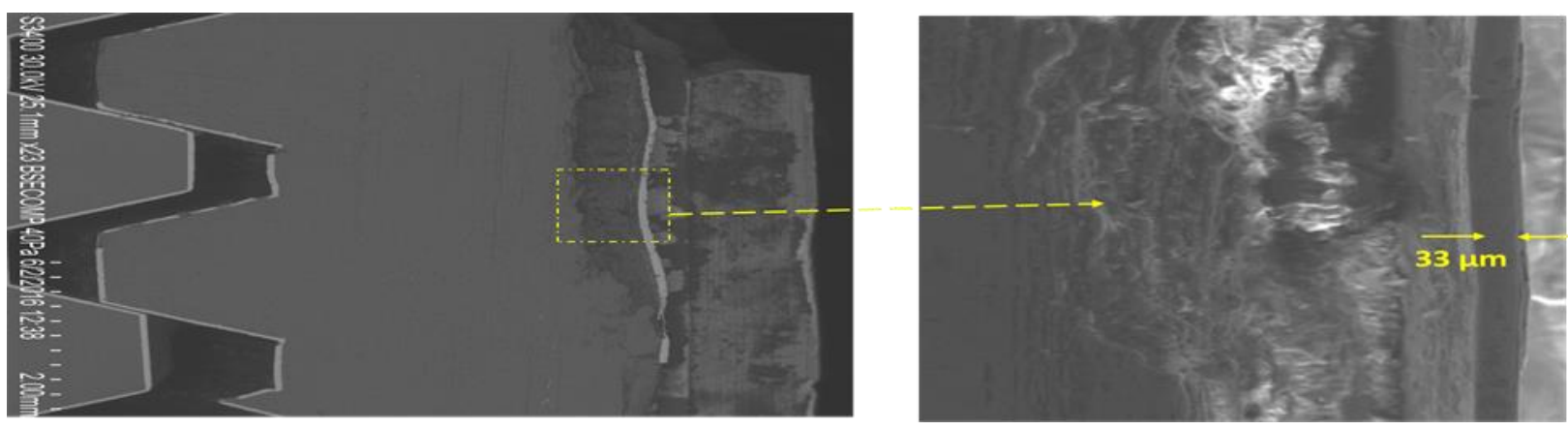

$\mathrm{ma}$

gni

fica

tio

n

sho

win

$\mathrm{g}$

sig

nifi

$\mathrm{t}$ erosion of coating.

Figure 2. SEM of Grade-B Ni-PTFE connector viewed from outer shell. The picture to the right is a higher magnification showing corrosion occurring under the coating with $33 \mu \mathrm{m}$ of the remaining coating.

Figure 3. SEM (3a) Remaining layers of Grade-B Ni-PTFE connector after 750 hours salt fog. SEM (3b) Remaining layer of Grade-A Ni-PTFE connector after 500 hours salt fog.

Table 1. Comparison of the measured thickness of the layers in each grade of connectors.

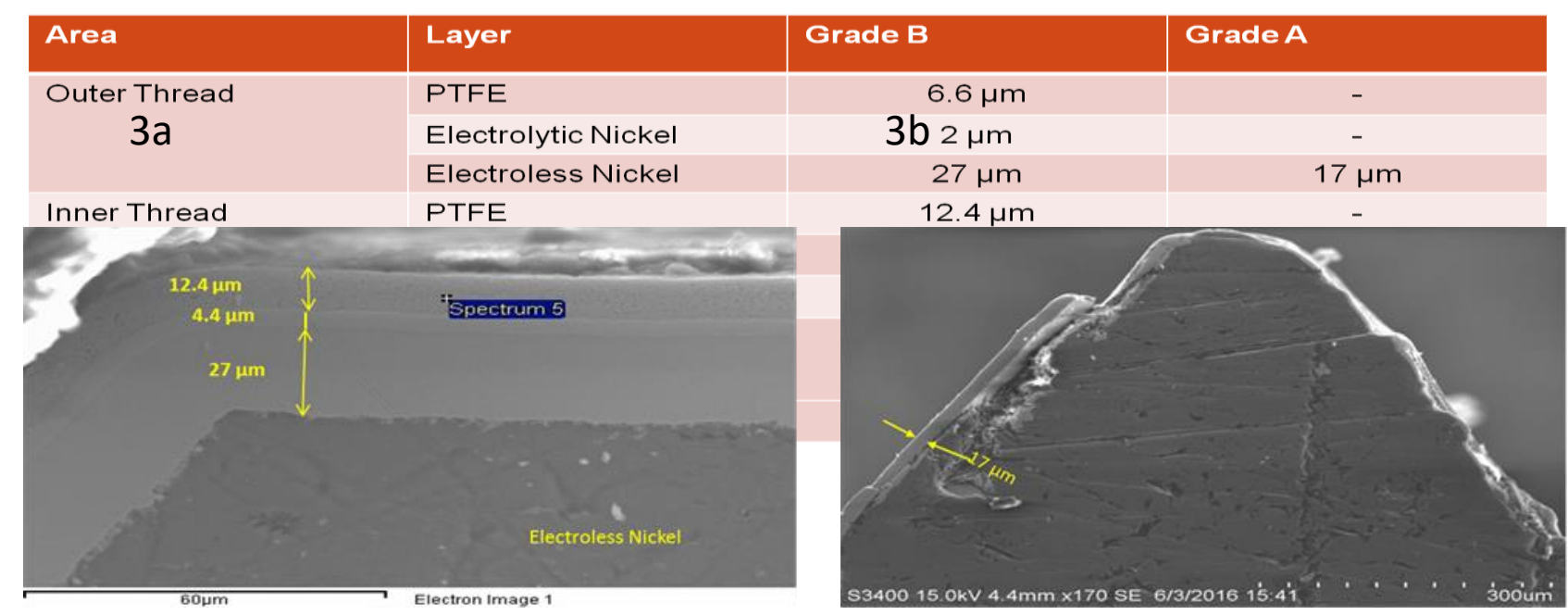

